\title{
Two Dimensional Computer Simulation of Plasma Immersion Ion Implantation
}

\author{
K. G. Kostov ${ }^{1}$, J. J. Barroso, and M. Ueda \\ Associated Laboratory of Plasma - LAP, National Institute for Space Research - INPE \\ Av. dos Astronautas 1758, C.P. 515, CEP 12201 970, São José dos Campos, SP, Brazil \\ ${ }^{1}$ Department of Physics and Chemistry, Engineering Faculty of Guaratinguetá FEG, UNESP \\ Av. Ariberto Perreira da Cunha 333, Guaratinguetá, SP, Brasil
}

Received on 30 January, 2004; revised version received on 6 May, 2004

\begin{abstract}
The biggest advantage of plasma immersion ion implantation (PIII) is the capability of treating objects with irregular geometry without complex manipulation of the target holder. The effectiveness of this approach relies on the uniformity of the incident ion dose. Unfortunately, perfect dose uniformity is usually difficult to achieve when treating samples of complex shape. The problems arise from the non-uniform plasma density and expansion of plasma sheath. A particle-in-cell computer simulation is used to study the time-dependent evolution of the plasma sheath surrounding two-dimensional objects during process of plasma immersion ion implantation. Before starting the implantation phase, steady-state nitrogen plasma is established inside the simulation volume by using ionization of gas precursor with primary electrons. The plasma self-consistently evolves to a non-uniform density distribution, which is used as initial density distribution for the implantation phase. As a result, we can obtain a more realistic description of the plasma sheath expansion and dynamics. Ion current density on the target, average impact energy, and trajectories of the implanted ions were calculated for three geometrical shapes. Large deviations from the uniform dose distribution have been observed for targets with irregular shapes. In addition, effect of secondary electron emission has been included in our simulation and no qualitative modifications to the sheath dynamics have been noticed. However, the energetic secondary electrons change drastically the plasma net balance and also pose significant X-ray hazard. Finally, an axial magnetic field has been added to the calculations and the possibility for magnetic insulation of secondary electrons has been proven.
\end{abstract}

\section{Introduction}

The implantation of ions into metallic surfaces is an important industrial process performed on such diverse products as cutting tools, stamping tools, draw dies, precision ball bearings, orthopedic devices, automotive parts and aerospace components. Ion implantation creates a thin surface layer of modified material, resulting in increased hardness, fatigue life, and corrosion resistance; reduced wear, sliding friction and galling, and modified electrical and optical properties [1]. Conventional ion implantation is commonly performed by raster scanning of an accelerator ion beam over the target. Typical drawbacks of this procedure, such as small spot size, low ion current, high production costs etc., can be avoided or reduced significantly in Plasma Immersion Ion Implantation (PIII) [2]. In PIII the target is immersed in weakly ionized plasma and pulsed to a high negative potential (up to $-100 \mathrm{kV}$ ). When the high voltage pulse is applied, nearly instantaneously $\left(\backsim \omega_{p e}^{-1}\right)$ electrons in the plasma are driven away to uncover a region of uniform ion density called "matrix ion sheath". Then, ions from the sheath are accelerated toward the target and implanted into the workpiece surface. To maintain the ion flux, the sheath edge will expand outwards uncovering more ions until the end of the voltage pulse. This sequence is repeated many times with a chosen repetition frequency. As a result of PIII processing a high implantation dose at low cost can be achieved. This method has an advantage over conventional ion beam implantation in that it is non line-of-sight, so beam rastering and target manipulation are not necessary. Also target sputtering is reduced, since ions are implanted perpendicular to the surface. PIII is widely used both in metallurgical and semiconductor processing and more recently for treatment of dielectric samples.

In this paper, the powerful numerical technique of particle-in-cell (PIC) simulation has been used to study sheath formation and dynamics of PIII. Two-dimensional cylindrical simulations permit us to investigate the process when sheath dimensions are large compared to the typical scale of the implanted object, where conformality is not assured. We consider three important issues, which should be addressed before wide-scale commercialization of PIII: (1) implant conformality; (2) secondary electron emission and (3) concept of magnetically insulated PIII. This article is organized as follows: A short introduction is followed by Section II where a brief review of theoretical models of sheath formation and dynamics is presented. The details of computer modeling of PIII using particle-in-cell (PIC) code KARAT 
are described in Section III. In next section, the implant conformality for PIII of complex shaped samples as well as the effects of secondary electron emission and external magnetic field are addressed. Finally, Section V summarizes the simulation results and outlines perspectives for future work.

\section{Plasma Sheath Dynamics}

In contrast to conventional beam line ion implantation, the current of the implanted ions cannot be measured reliably in PIII. However, for technological application information about the ion flux, the angle of incidence and the energy distribution of the implanted ions must be available. All these parameters are determined by the dynamics of plasma sheath and thus a series of models for PIII process was developed and published [3]-[7].

The analytical models of PIII are usually based on a simplified 1D pilot model presented by Lieberman [3]. This model was subsequently extended to account for cylindrical and spherical geometries, for collisions or finite pulse rise time, yet all present theoretical models are limited to one dimension [4]-[7]. In brief, following application of the pulsed negative voltage $V$, plasma electrons are rapidly repelled (in a few ns) to uncover a region of uniform ion density. This region is known as the ion matrix sheath, and has a thickness $s_{0}$ given by

$$
s_{0}=\sqrt{\frac{2 \varepsilon_{0} V}{e n_{0}}}
$$

where $n_{0}$ is the initial plasma density, $\varepsilon_{0}$ is the electric permittivity of free space, and $e$ is the elemental charge.

On a longer time scale $(\sim \mu s)$ ions are accelerated across the sheath by the applied electric field and are driven into the target surface. As ions are implanted, charge imbalance repels more electrons away form the workpiece, thereby uncovering more ions to be implanted. The time-dependent sheath thickness is given by [3]

$$
s=s_{0}\left(\frac{2}{3} \omega_{p i} t+1\right)^{1 / 3}
$$

here $\omega_{p i}=\left(n_{0} e^{2} / \varepsilon_{0} M\right)^{1 / 2}, M$ is the ion mass, and $t$ is the time into the PIII pulse.

Subsequently the sheath expands until a steady-state Child-Langmuir sheath is formed and in this case the implantation current density is given by the " $3 / 2$ power" law which for planar geometry is

$$
j_{i}(t)=\frac{4 \varepsilon_{0}}{9} \sqrt{\frac{2 e}{M}} \frac{V^{3 / 2}}{[s(t)]^{2}}
$$

Further insight into plasma sheath dynamics is gained from 1D computer simulations, which are in a relatively good agreement with the theoretical models [7]-[10]. However, issues related to evolution of the sheath shape cannot be addressed with 1D techniques. Multidimensional calculations are needed to study such phenomena as plasma transient sheaths and their evolution.
The most attractive feature of PIII is its possibility of homogeneous and conformal implantation in threedimensional structures [2]. However, in practice, a conformal implantation in 3D sample is not a priori guaranteed. The complicated behavior of the expanding plasma sheath around complex shaped targets leads to concentration and depth profile inhomogeneities. Experimental study [11] have shown that large deviations in the retained dose could be observed in dependence on the sample geometry and plasma sheath thickness. Experiments for determining the dose distribution are very complicated so that various computer programs for simulating the implantation were developed [8]-[10],[12]-[17]. A self-consistent 3D calculation of the sheath dynamics and ion current is prohibitively time-consuming. That is why, 2D PIC simulations [12]-[15] were employed to investigate time-dependent formation of the sheath, depletion of the initial ion matrix sheath, and the slow expansion of the sheath into the ambient plasma. Aside from the implant conformality, another key issue related to PIII that can be investigated by 2D PIC simulations, is the role of secondary electrons. As each ion is implanted, electrons are liberated from the target and are rapidly accelerated through the sheath potential. For most metallurgical applications the secondary emission yield is quite large, often ranging between 3-20 [16]. Therefore uncontrolled secondary emission could significantly reduce the PIII efficiency to as low as $5 \%$. Furthermore, the Bremsstrahlung X-rays produced by energetic secondary electron bombardment of the chamber wall pose a potential safety hazard. The suppression of secondaries remains an open question and virtually all PIII devices are currently operated without any secondary electron control. One proposed (but untested) technique for suppressing secondary electrons [17] uses an external axial magnetic field. Secondary electrons are trapped near the workpiece surface, where the local electric field is substantially decreased by the space charge. Then subsequent electrons, which are emitted from the workpiece surface can be reabsorbed by the target. The magnitude of magnetic field is chosen so that the secondary electrons are strongly confined, while ion motion is only slightly perturbed. Inclusion of an axial external magnetic field leads to significant changes in the plasma net balance and the PIC computer simulations can provide further insight into this.

The numerical calculations, reported in this article, are based on creation of a realistic, self-consistent, 2-D, PIII simulation, where steady-state plasma is generated inside a vacuum chamber by ionization of gas precursor. In all previous 2-D computer simulations, a simplified picture of the plasma before implantation phase was assumed: ions and electrons extend with uniform density throughout the plasma to the wall. Thus, the structure of the sheath in front of the target is neglected, and so are the gradients in particle density and particle drift motion toward the wall. In contrast to the previous simulations that start with homogeneous, cold plasmas, in our simulation the plasma is generated by ionization of background gas, so that the plasma density self-consistently grows up and adapts itself to the vacuum vessel. Then, at a certain simulation time, when the desired initial plasma density is achieved, the implantation phase is 
initiated by turning on the target bias. In a realistic PIII experiment, the voltage pulse rise and fall times may constitute a significant fraction of the total pulse width. That is why, we simulated a voltage pulse with finite rise and fall times. During the implantation phase ions are implanted into the target but in the same time new charged particles are created by ionization. So we will be able to maintain constant average plasma density inside de vacuum chamber during sufficiently long voltage pulse. Another advantage of this approach is that the plasma generated by ionization is continuously replenished while in case of initial fixed plasma density the plasma will be gradually depleted due to the ion implantation.

The main objectives of our numerical study can be summarized as follow:

1. Obtaining improved description of the ion current density on the target, the matrix sheath expansion and dynamics by a realistic PIII simulation where equilibrium plasma is generated by ionization of neutral gas precursor that fills the vacuum chamber.

2. Detailed study of implant conformality in case of complex shaped targets

3. Examining the role of secondary electrons for the electron/ion balance and the plasma sheath dynamics

4. Investigation of the effectiveness of an externally applied magnetic field for suppressing secondary electron emission

\section{Description of the Numerical Algo- rithm}

Computer simulation has become a powerful tool for the study of plasma physics. Numerical simulation of plasmas is achieved by simply computing the motion of a collection of charged particles, interacting with each other and with external applied fields. When appropriate methods are used, relatively small systems of thousand "super-particles" can indeed simulate accurately the collective behavior of real plasmas. The development of new algorithms and the availability of more powerful computers has allowed particle simulations to progress from simple one-dimensional, electrostatic problems to more complex and realistic situations involving electromagnetic fields in multiple dimensions and up to $10^{6}$ super-particles.

Any computer simulation - one, two or threedimensional starts with some initial particle distribution, i.e. the particle positions and velocities are known. First, the electromagnetic fields are calculated from Maxwell's equations and the forces on the particles are found using the electric and magnetic fields in Newton-Lorentz equation of motion. Then the particles are moved in a small distance and the fields due to the new particle positions and velocities are recalculated. This procedure is repeated for many time steps. The difference from laboratory plasmas is that simulations proceed discontinuously in time, step by step, using digital computation. We use a spatial grid on which the fields are calculated and a temporal grid, which is sufficiently fine to follow the plasma evolution with acceptable preci- sion. Currently various computer codes are available for simulation of plasma that use the above described algorithm commonly refereed to as particle-in-cell (PIC) method.

Computer simulations were performed using code KARAT [18]. It is a fully electromagnetic, relativistic, particlein-cell (PIC) code, which has proven its usefulness in many fields of plasma physics, microwave electronics, electron beam dynamics, etc. A finite difference scheme with overstepping on rectangular shearing grid is used to solve Maxwell's equations with fulfillment of complex boundary conditions. Plasma is simulated either by macro-particles or by a linear model. If necessary, inelastic processes such as secondary electron emission form surfaces, gas ionization and recombination can be included. Depending on the simulated problem geometry one can use 1D, 2D or 3D KARAT code version. In our simulation we utilize a two dimensional electrostatic version of KARAT. This means that assuming azimuthal symmetry only two space variables ( $\mathrm{r}$ and $\mathrm{z}$ in cylindrical geometry) are considered while three component of velocity $\mathrm{v}_{z}, \mathrm{v}_{r}$ and $\mathrm{v}_{\theta}$ are retained (this combination of parameters is usually called $2.5 \mathrm{D}$ version). Electric field components Er and Ez are calculated by solving the Poisson's equation. The particles are moved by the combined action of the electric field and an externally applied, user specified, static magnetic field in axial direction. The new particles positions and velocities are obtained by solving the equation of motion.

Layout of the PIII system geometry simulated with KARAT is shown in Fig. 1. Dimensions of $13-\mathrm{cm}$ radius, 38$\mathrm{cm}$ long vacuum camera have been chosen closely to those of the experimental PIII facility operated at LAP/INPE [19]. The vacuum chamber wall is grounded while a thin insulated sample holder is located at the camera axis. A target to be implanted with radius of $1.5 \mathrm{~cm}$ is placed at the end of the sample holder and during the implantation phase the holder and the sample are biased at $-10 \mathrm{kV}$. Detailed contour plot of the equipotential lines around the sample is shown in Fig. 1.

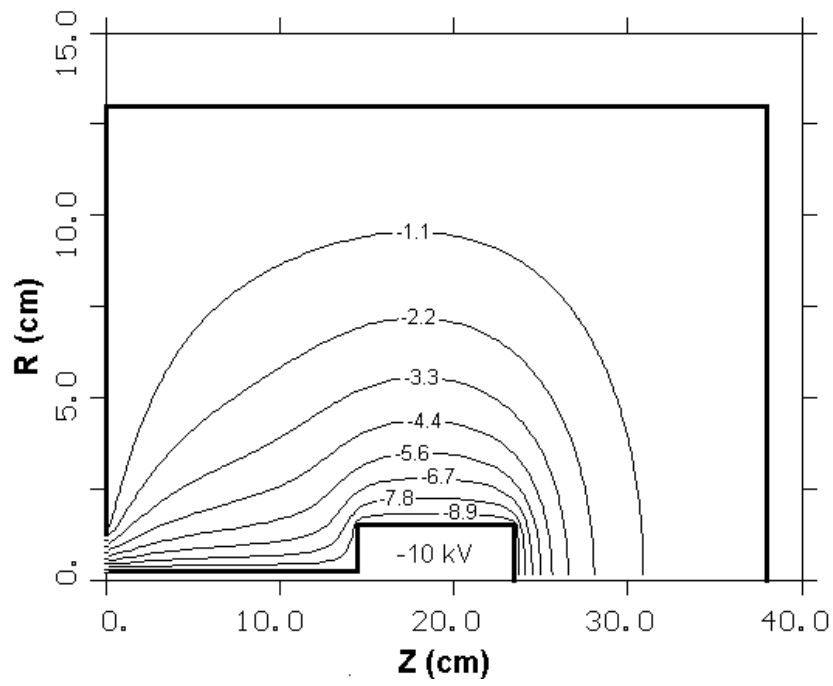

Figure 1. Configuration of PIII simulation setup with $-10 \mathrm{kV}$ target bias. Contour plot of equipotential lines around the target is presented. 


\section{Simulation Results}

The simulation begins at time $\mathrm{t}=0.0 \mathrm{~s}$ with a vacuum vessel filled with nitrogen gas (concentration on the order of $10^{14}$ $\mathrm{cm}^{-3}$ ) and high voltage pulse off. During the first $1.25 \mu \mathrm{s}$, nitrogen plasma $\left(\mathrm{N}_{2}^{+}\right.$ions) is gradually generated inside the simulation volume by ionization of the neutral gas molecules using primary electrons injected from the camera wall. Real mass ratios were employed. Probabilistic Monte-Carlo collision algorithm is applied to simulate the ionization process. By adjusting the background gas and the primary electron current concentration (both code input parameters), a desired plasma density can be achieved. Then for the time interval from $\mathrm{t}=1.25 \mu \mathrm{s}$ to $\mathrm{t}=1.5 \mu \mathrm{s}$, the sample bias potential is linearly increased from zero to $-10 \mathrm{kV}$. After that, the computer simulation proceeds with high voltage pulse on. By using this procedure, steady-state plasma with density on the order of $10^{9} \mathrm{~cm}^{-3}$ and temperature about $3 \mathrm{eV}$ is obtained and maintained in the vacuum chamber. These plasma parameters are close to the experimentally measured plasma temperature and density [20]. In our calculations square cell with size $\Delta \mathrm{z}=0.0040-0.0045 \mathrm{~m}$ and $\Delta \mathrm{r}=0.0013$ $0.0015 \mathrm{~m}$, and a time step of $2 \times 10^{-12}$ s were typically used. Total number of the super-particles, representing the plasma in the simulation, is around 140000. Fig. 2 shows the number of each kind of plasma particles used in our simulation as a function of time. As one can see from Fig. 2 the net charge balance is maintained in the time which allows a detailed investigation of sheath temporal evolution.

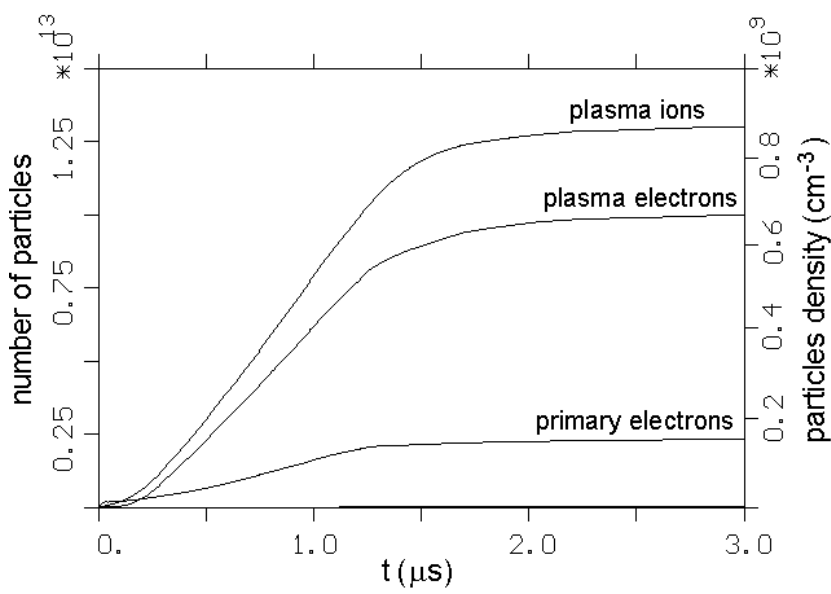

Figure 2. Plasma particles net balance.

After application of high voltage pulse, plasma electrons are repealed away from the target leaving behind ion matrix sheath. Fig. 3, depicts the configuration space of the plasma electrons at the time $\mathrm{t}=3.0 \mu \mathrm{s}$ showing the position of and the shape of the plasma sheath at that moment.

Plasma ions are accelerated across the sheath and are implanted into the sample surface by the applied electric field. Fig. 4 shows the energy distribution of the implanted ions at simulation time $\mathrm{t}=3.0 \mu \mathrm{s}$. The incident ions have almost monoenergetic energy distribution centered at about $9.25 \mathrm{keV}$. Since ion transit time across the sheath is quite large (on the order of $1 / \omega_{p i}$, during ion flight across the sheath it manages to expand, in that way changing both the potential barrier shape and magnitude, so that the implanted ions do not get the full bias voltage [8].

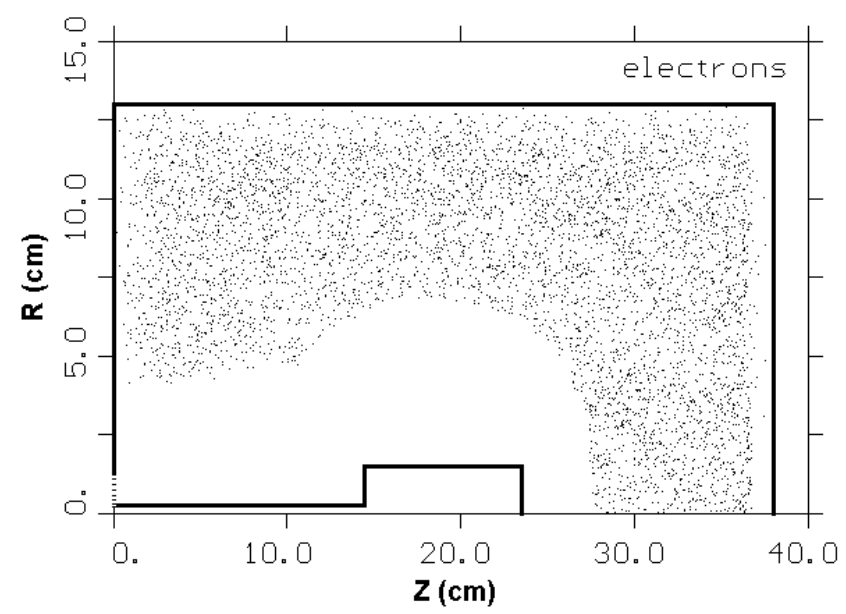

Figure 3. Snapshot showing the electron distribution at $3.0 \mu$ s with the target charged to $-10 \mathrm{kV}$.

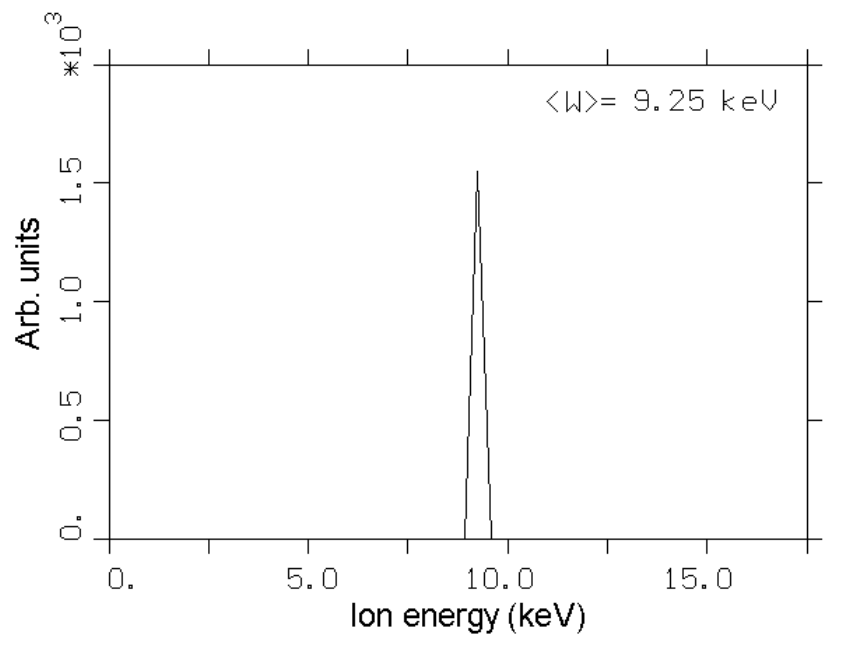

Figure 4. Energy distribution of the incident ions.

\subsection{Implant conformality}

In contrast to $1 \mathrm{D}$ simulations, 2D computer simulations can provide information about the current spatial distribution. We considered three targets with different geometry - regular shape sample, convex shape sample and concave shape sample. The electric field lines, the typical ion trajectories and the axial distributions of implantation current (actually implantation current per unit length in cylindrical symmetry) for these three different targets are shown in Fig. 5. 

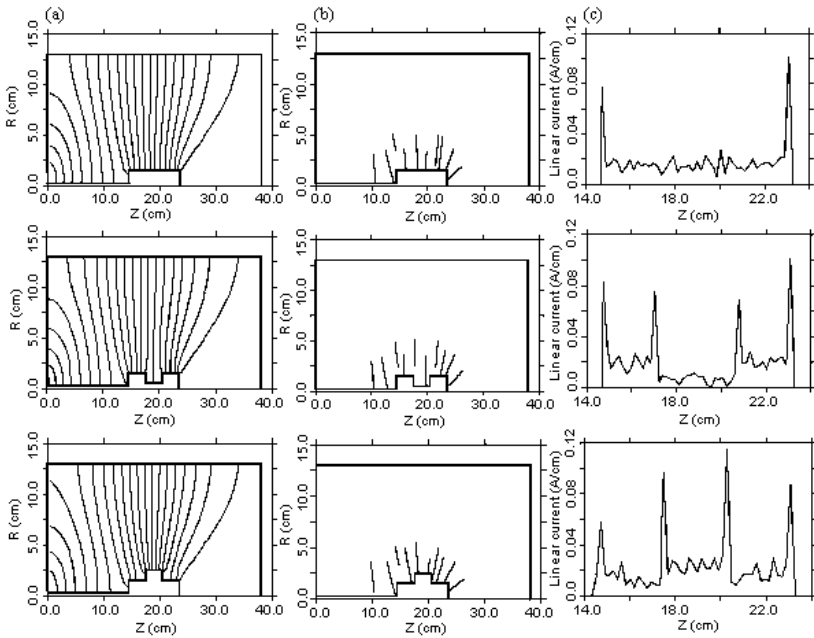

Figure 5. Particle-in-cell simulation of PIII into targets with different geometry - regular shape sample, concave shape sample and convex shape sample. Comparison of a) electric field lines, b) typical ion trajectories and c) average implanted current density.

In case of target with regular geometry, one can see that aside from some fringing fields that appear at the sample edges, the electric field at the workpiece surface is homogeneous. Thus, the implantation current density is also approximately uniform along the sample, except for the spikes located at the sample edges where more ions are implanted due to the local electric field enhancement. The tiny peaks that appear in the current distribution are result of the numerical algorithm. By using finer space grid and more super particles to represent the plasma, one can obtain smoother distribution, however this will lead to excessive computation time. In our simulations for solving the Poisson's equation, we usually employed a space grid $1.3 \times 4 \mathrm{~mm}$ and a time step of $2.0 \mathrm{ps}$ for solving the equation of motion. The plasma was represented by 130000-140000 super-particles. With these numerical parameters, one computer simulation typically ran for 8-10 days using Pentium 4, 2.0GHz computer. For this reason a compromise between the computation time and the accuracy should be made. The basic plasma parameters obtained in the simulation (plasma temperature $\backsim 3.0 \mathrm{eV}$, plasma density $\backsim 10^{9} \mathrm{~cm}^{-3}$, transient plasma sheath size $\backsim 6.0 \mathrm{~cm}$, implantation current time profile etc.) are in good agreement with the experimental measurements in [20]. So, despite some roughness of the distribution functions, the computer simulation with the above selected numerical parameters represents well the qualitative behavior of expanding sheath and PIII process. A series of simulations with higher resolution for a smaller chamber are prepared, but the calculations have yet not been performed.

Now, looking at the simulation results for complexshaped targets, one can see that the electric field at the sample surface is no longer homogeneous. There is a concentration of the electric field lines at the sample edges and high points while the electric filed is weakened at the sample holes and trenches. Fig. 5c shows that for complex shaped samples the implantation current along the target is nonuniform. Also, from the ion trajectories presented in Fig. 5b, one can infer that concave surface receive a smaller dose compared to that of convex surface. Generally speaking, more ions are implanted into the topmost part (edges, peaks, crests etc.) of a complex shaped sample, while fewer ions will reach the lowermost sample parts such as holes, bores, gaps and other low points. As a result the implantation current density along samples with irregular geometry is nonuniform. So, the irregular sample geometry will result in spatial inhomogeneity of the implanted current. This geometry effect should be accounted for, especially, in case of implantation of semiconductor devices.

For both irregular target shapes, the influence of the geometry on the average energy of the incident ions was investigated. No effect of sample shape on the average implantation energy was found. However, the impact angle is influenced by the sample geometry and a detailed study of this effect is under way.

\subsection{Secondary electron emission}

Secondary electron emission caused by incident ions can have a severe effect on the PIII process. For $\mathrm{N}_{2}^{+}$ions, depending on the bias potential and target material, between 3 and 20 electrons can be emitted for every implanted ion [16]. This causes both an enormous shunt current, and hence a power drain, and significant X-ray emission, when the accelerated secondary electrons hit the chamber wall. A series of simulations were conducted to study the secondary emission process and its influence on sheath dynamics. The secondary electron yield was chosen as four electrons per each implanted ion. The addition of secondary electrons resulted in no qualitative modifications to the sheath formation and expansion. However, an important effect of the secondary electrons is the alteration of the net electron/ion balance as can be seen in Fig. 6. Energetic secondaries can ionize the background gas leading to an increase of plasma density. That is way to maintain same plasma concentration, as in previous simulations ( $\backsim 10^{9} \mathrm{~cm}^{-3}$ as shown in Fig. 2), we needed to decrease the number of injected primary electrons. This effect, that the secondary electrons help to sustain an electric discharge is well known. However the uncontrolled secondary emission can significantly reduce the PIII efficiency and also pose the hazard of Bremsstrahlung X-rays emission.

The effect of secondary electron bombardment of the chamber can be seen in Fig. 7 which plots the energy distribution of all electrons arriving at the vacuum camera wall. Along with the low energy plasma electrons, the distribution exhibits a high-energy peak around $10 \mathrm{keV}$ associated with energetic secondary electrons. The current distribution of the electron current is shown in Fig. 8. The broad maximum at the center of the distribution is caused by secondary electrons. They leave the workpiece surface and are accelerated radially outward by the sheath potential until they hit the camera wall. 


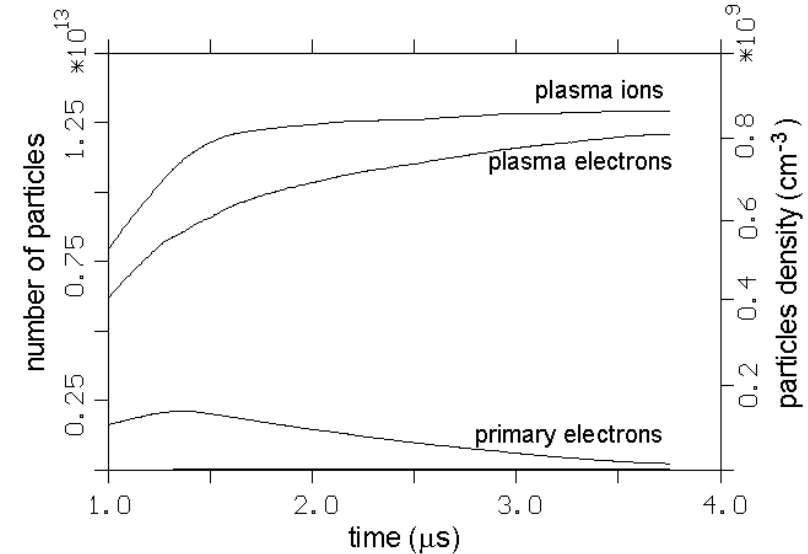

Figure 6. Plasma particle balance with inclusion of secondary electron emission.

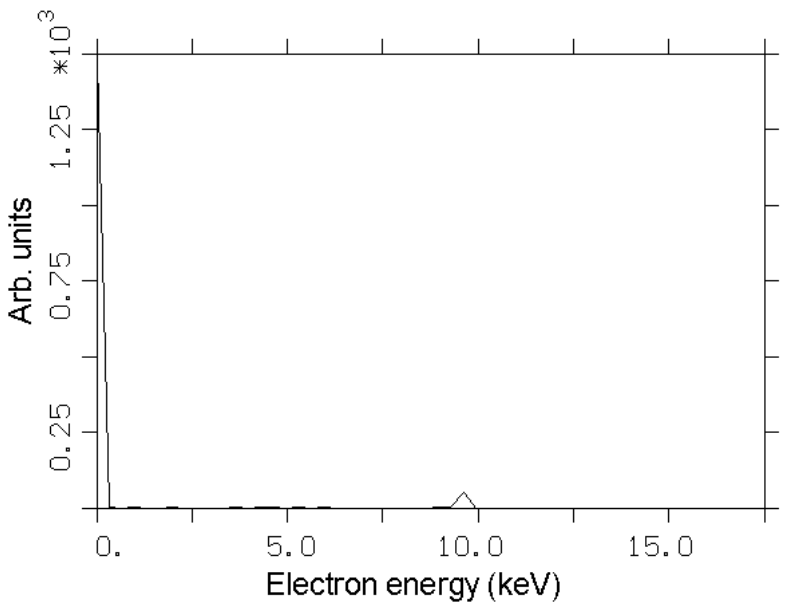

Figure 7. Energy distribution of the electrons impinging the vacuum camera wall.

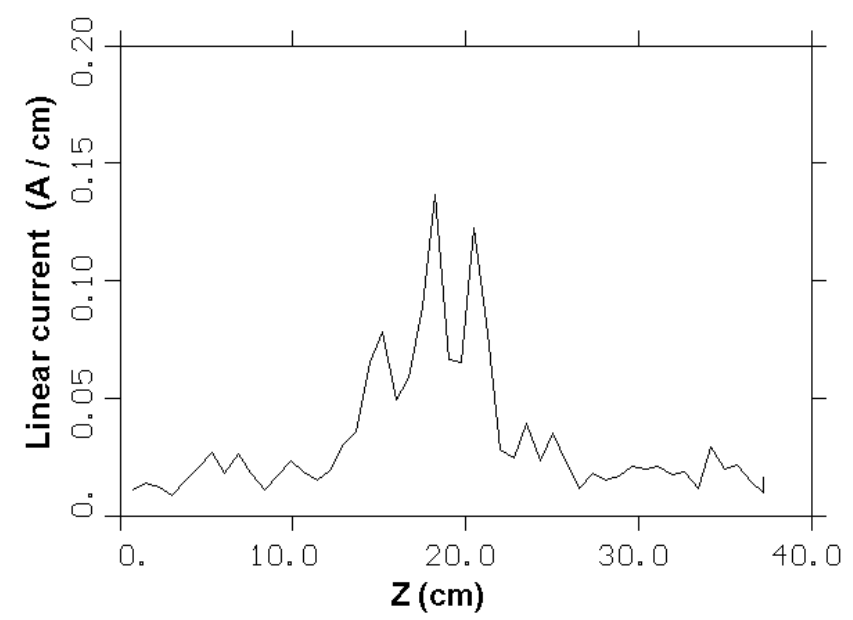

Figure 8. Current distribution of the electron current at the vacuum camera wall.

\subsection{Magnetic insulation of secondary elec- trons}

In our simulations, we have investigated a possibility for controlling the secondary electrons by an externally applied axial magnetic field. Also it is an interesting question to study the dynamics of a magnetized plasma sheath. In our simulation a target with radius $1.5 \mathrm{~cm}, 5-\mathrm{cm}$ length and biased at $-10 \mathrm{kV}$, is immersed in uniform magnetic field $\mathrm{B}=\mathrm{B}_{z}$. The secondary electrons emerge from the workpiece at finite energy of a few eV and under combined action of electric field $\mathrm{E}=-\mathrm{E}_{r}$ and the axial magnetic field move following cycloidal trajectories in the $r-\theta$ plane. Therefore the secondary electrons are confined in a layer near to the target surface. Fig. 9 shows 3D plot of electron density at the simulation time $\mathrm{t}=2.0 \mu \mathrm{s}$. From this figure one can see the ambient plasma, which extends through the entire simulation region with homogeneous average plasma density. Next, the sheath is presented by a space without electrons and finally the magnetically insulated secondary electrons are shown as bunches confined in a small region near to the sample surface. The magnitude of magnetic field $\mathrm{B}=0.04 \mathrm{~T}$ has been selected in such a way, that the secondary electrons are strongly confined in the target vicinity while ion motion is only slightly perturbed. This is confirmed by the ion trajectories that practically do not differ from the ones observed in case without magnetic field. Also, the fact that ions are not perturbed by the magnetic field applied in the simulation has been confirmed by the axial distribution of the ion current and the average ion energy. They both are virtually the same as in the case with magnetic field.

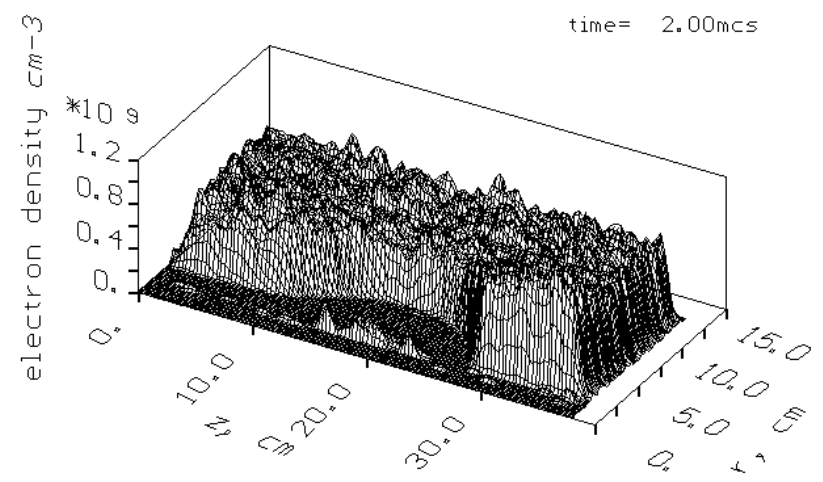

Figure 9. 3D plot of the electron density showing magnetic confinement of the secondary electrons near to the target surface.

Emitted secondary electrons are effectively confined near to the target. They form an electron layer reducing the local electric field, which can lead to further secondaries being reabsorbed by the sample. Eventually, the electrons can diffuse away axially (see Fig. 9), however there is no energetic electron bombardment of the wall and no potential risk for producing of X-rays.

The effect of magnetic field inclusion on the plasma net balance can be observed in Fig. 10, which compared to Fig. 6 , demonstrates that the balance of plasma particles is significantly altered when magnetic field is applied. Since the secondary electrons are now confined near to the target surface, they can not ionize the neutral gas and consequently do not play any role in plasma net balance. Our simulation showed that the dynamics of magnetized plasma sheath was considerably more complex, that the one without magnetic 
field. As it is shown in a recent study [21] an external magnetic field applied in PIII can lead to some favorable effects, so that the simulation of this effect is an important issue.

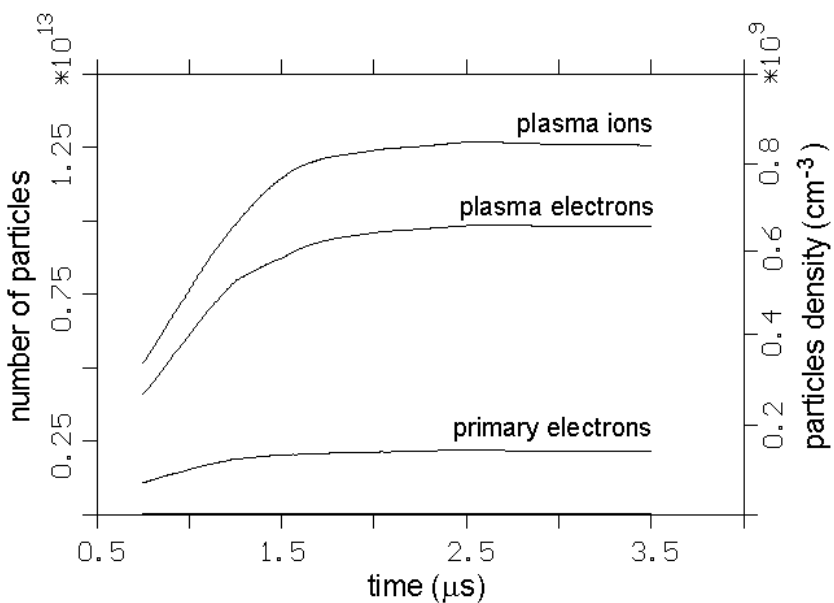

Figure 10. Effect of axial magnetic field $\mathrm{B}_{z}=0.04 \mathrm{~T}$ on the net particle balance in case of constant secondary electron emission yield $\gamma=4$.

\section{Conclusions}

The appeal of plasma immersion ion implantation is that it enables the well-known benefits of ion implantation to complex shapes in a quick and cost-effective manner. However, to take full advantage of non-line-of-sight property of PIII, it is important to ensure a uniform implantation. We have employed 2D PIC computer simulation to study PIII for a variety of different implantation morphologies. Steady state nitrogen $\mathrm{N}_{2}^{+}$plasma has been generated into the vacuum chamber by ionization of neutral gas with primary electrons. This allows creating realistic computer simulation, which closely approaches to the real experiment. The studies have been confined to applied voltage of $-10 \mathrm{kV}$ and plasma density on the order of $10^{9} \mathrm{~cm}^{-3}$. The dependence of ion current density on the target geometry has been investigated. The ion trajectories converge over topmost parts of the sample (ridges, edges, peaks etc.) and diverge near the lowest sample points such as holes, bores, trenches and other low points. Substantial secondary emission often accompanies PIII limiting the electrical system efficiency and also leading to soft X-ray production. Secondary electron emission has been included in the simulation and has been found to play a significant role in a plasma net balance. Finally, an external magnetic field has been added in the simulation to examine the concept of magnetic insulation of the secondary electrons. The magnitude of magnetic field has been chosen so that the secondary electron trajectories are greatly altered while ion motion remains virtually unperturbed. Secondary electrons are trapped near the workpiece surface decreasing substantially the X-ray hazard. Magnetized sheath motion has been found to be considerably more complex and additional simulations are planned in a near future.

\section{Acknowledgments}

This work is supported by the Fundação de Amparo à Pesquisa do Estado de São Paulo (FAPESP). One of the authors, K.G. Kostov, wishes to thank FAPESP for a visiting researcher scholarship, grant 02/08486-5.

\section{References}

[1] M. Nastasi and J. W. Meyer, Ion-Solid Interections: Fundamentals and Applications, Cambridge University Press, Cambridge, (1996).

[2] J. R. Conrad, J. L. Radtke, R. A. Dodd, F. J. Worzala, and N. C. Tran, J. Appl. Phys. 62, 4591 (1987).

[3] M. A. Lieberman, J. Appl. Phys. 66, 2926 (1989).

[4] J. T. Scheuer, M. Shamin, and J. R. Conrad, J. Appl. Phys. 67, 1241 (1990).

[5] V. Vahedi, M. A., M. A. Lieberman, M. A. Alves, P. P. Verboncoeur, and C. K. Birdsall, J. Appl. Phys. 69, 2008 (1991).

[6] R. A. Steward and M. A. Lieberman, J. Appl. Phys. 70, 3481 (1991).

[7] K. U. Riemann and Th. Daube, J. Appl. Phys. 86, 1202 (1999).

[8] B. P. Wood, J. Appl. Phys. 73, 4770 (1993).

[9] B. Briehl and H. M. Urbassek, Surf. Coat. Technol. 131-135, 156, (2001).

[10] D. T. K. Kwok, M. M. Bilek, D. R. McKenzie, and P. K. Chu, Appl. Phys. Lett. 82, 1827 (2003).

[11] P. Huber, G. Keller, J. W. Gerlach, S. Mandl, W. Assmann, and B. Rauschenbach, Nucl. Instr. Meth. B, 161-163, 1085 (2000).

[12] R. Faehl, B. Volder, B. Wood, J. Vac. Sci. Technol. B12, 884 (1994).

[13] D. J. Rej, R. J. Faehl, J. N. Matossian, Surf. Coat. Technol. 96, 45 (1997)

[14] G. Keller, S. Mandl, U. Rude, B. Rauschenbach, Surf. Coat. Technol. 136, 117 (2001).

[15] B. Rauschenbach and S. Mandl, Nucl. Instr. Meth. B, 206, 803 (2003).

[16] M. M. Shamin, J. T. Scheuer, R. P. Fetherston, and J. R. Conrad, J. Appl. Phys. 70, 4756 (1991).

[17] D. J. Rej, B. P. Wood, R. J. Faehl, H. H. Fleischmann, J. Vac. Technol. B 12, 861 (1994).

[18] V. P. Tarakanov User's Manual for Code KARAT, Berkeley, Research Associated, Berkeley, USA 1994.

[19] M. Ueda, L. A. Berni, G. F. Gomes, A. F. Beloto, E. Abramoff, and H. Reuther, J. Appl. Phys. 86, 4821 (1999).

[20] L. A. Berni, M. Ueda, G. F. Gomes, A. F. Beloto, and H. Reuther, J. Phys. D.: Appl. Phys. 33, 1592 (2000).

[21] D. L. Tang, R. K. Y. Fu, X. B. Tian, P. Peng, and P. K. Chu, Nucl. Instr. Meth. B, 206, 808 (2003) 